\title{
Body Composition Analysis of Staff members of College Using Bioelectrical Impedance Analysis Method
}

\author{
Swaroopa Rani N. Gupta
}

\begin{abstract}
Aims: In this analysis of body composition such as body weight, BMI, body fat percentage, segmental subcutaneous fat $\&$ skeletal muscle percentage (whole body, trunk, legs and arms), resting metabolism, visceral fat level and body age is done by bioelectrical impedance technique and results are interpreted and corresponding instructions for better health improvement is given.

Methods: Body composition analysis of teaching and nonteaching staff members of Brijlal Biyani Science College Amravati Maharashtra India using bioelectrical impedance analysis method is done. For ideal weight management and for a more accurate and precise body composition analysis full body sensing technology karada scan body composition monitor - HBF-375 is used. The general principle behind bioelectrical impedance analysis is that two or more conductors are attached to a person's body and a small electric current is sent through the body. The resistance between the conductors provides a measure of body fat between a pair of electrodes, since the resistance to electricity varies between adipose, muscular and skeletal tissue.

Result: Interpretation of body composition analysis report of Teaching Staff members (Senior College) shows that

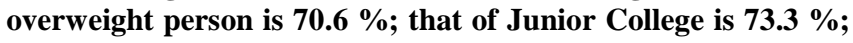
that of non-teaching staff members (laboratory) is $52.4 \%$ while; that of office is $76.9 \%$; and that of library is $100 \%$.
\end{abstract}

Index Terms-Body age, body fat, BMI, RM, skeletal muscle, subcutaneous fat, visceral fat.

\section{INTRODUCTION}

Bioelectrical impedance analysis (BIA) is a commonly used method for estimating body composition, and in particular body fat. Since the advent of the first commercially available devices in the mid-1980s the method has become popular owing to its ease of use, portability of the equipment and it's relatively low cost compared to some of the other methods of body composition analysis. It is familiar in the consumer market as a simple instrument for estimating body fat. BIA actually determines the electrical impedance, or opposition to the flow of an electric current through body tissues which can then be used to calculate an estimate of total body water (TBW). TBW can be used to estimate fat-free body mass and, by difference with body weight, body fat [1].

Many of the early research studies showed that BIA was quite variable and it was not regarded by many as providing an accurate measure of body composition. In recent years technological improvements have made BIA a more reliable and therefore more acceptable way of measuring body

Manscript received November 15, 2013; revised January 19, 2014

Swaroopa Rani N. Gupta is with the Department of Chemistry, Brijlal Biyani Science College Amravati, Maharashtra, India (email: swargupta@yahoo.com). composition. Nevertheless it is not a "gold standard" or reference method. Like all assessment tools, the result is only as good as the test done. Although the instruments are straightforward to use, careful attention to the method of use (as described by the manufacturer) should be given.

Simple devices to estimate body fat, often using BIA, are available to consumers as body fat meters. These instruments are generally regarded as being less accurate than those used clinically or in nutritional and medical practice. They tend to under-read body fat percentage [2].

Dehydration is a recognized factor affecting BIA measurements as it causes an increase in the body's electrical resistance, so has been measured to cause a $5 \mathrm{~kg}$ underestimation of fat-free mass i.e. an overestimation of body fat [3].

Body fat measurements are lower when measurements are taken shortly after consumption of a meal, causing a variation between highest and lowest readings of body fat percentage taken throughout the day of up to $9.9 \%$ [4].

Moderate exercise before BIA measurements lead to an overestimation of fat-free mass and an underestimation of body fat percentage due to reduced impedance [5]. For example moderate intensity exercise for 90-120 minutes before BIA measurements causes nearly a $12 \mathrm{~kg}$ overestimation of fat-free mass, i.e. body fat is significantly underestimated [6]. Therefore it's recommended not to perform BIA for several hours after moderate or high intensity exercise [7].

BIA is considered reasonably accurate for measuring groups, or for tracking body composition in an individual over a period of time, but is not considered sufficiently accurate for recording of single measurements of individuals [8].

The accuracy of consumer grade devices for measuring BIA has not been found to be sufficiently accurate for single measurement use and is better suited for use to measure changes in body composition over time for individuals [9].

Bioelectrical impedance analysis (BIA) is widely used in clinics and research to measure body composition. However, the results of BIA validation with reference methods are contradictory, and few data are available on the influence of adiposity on the measurement of body composition by BIA. BIA is a good alternative for estimating \%BF when subjects are within a normal body fat range. BIA tends to overestimate $\% \mathrm{BF}$ in lean subjects and underestimate $\% \mathrm{BF}$ in obese subjects [10].

Bioelectrical impedance analysis (BIA) is a promising tool in the evaluation of body composition in large population studies because it is fast, is inexpensive, and does not require extensive operator training or crossvalidation. The empiric nature of the relation between 
resistance and reactance measured by BIA and body composition has led to the development of equations that translate the raw data into liters of body water or kilograms of fat-free mass (FFM) or fat mass. These equations may not be easily transferred from one population to another if the populations differ significantly in important determinants of body composition such as age, obesity, and illness. Review of two recent studies from the Framingham Heart Study in which BIA was first compared with dualenergy X-ray absorptiometry (DXA) as a validation technique, and then compared with the body mass index (BMI, in $\mathrm{kg} / \mathrm{m} 2$ ) as an alternative estimate of body fat. BIA was a good predictor of DXA-derived FFM ( $r=0.85-0.88$, $P<0.001)$ and was superior to BMI as an estimator of body fat [11].

Over the past decade, considerable attention has been paid to accurately measuring body composition in diverse populations. Recently, the use of air-displacement plethysmography (AP) was proposed as an accurate, comfortable, and accessible method of body-composition analysis. AP is an accurate method for assessing body composition in healthy adults. Future studies should assess further the cause of the individual variations with this new method [12].

Obesity continues increasing at epidemic levels worldwide, as does the number of genetic studies that focus on obesity. Body mass index (BMI) is often used to characterize weight phenotypes and obesity status due to its simplicity. Refined measurements of body composition may be needed to understand variations in gene expression. This study explores gene expression when individuals are characterized as overweight based on BMI versus body fat percent. Individuals were recruited to a natural history protocol at the National Institutes of Health. Twelve Caucasian participants with the highest and lowest BMI were included. Whole-body air displacement plethysmography was performed to calculate body fat percent, and BMI was calculated. Fasting whole blood was collected and RNA extracted. Quantitative real time PCR array was used to determine expression of 96 obesity related genes. The PCR array from participants with high BMI compared to low BMI showed dysregulation of four genes: peroxisome proliferator-activated receptor gamma coactivator 1-alpha (PPARGC1A), pro-opiomelanocortin $(P O M C)$, growth hormone secretagogue receptor (GHSR), and leptin $(L E P)$, whereas participants with high body fat compared to low body fat showed dysregulation of one gene: PPARGC1A. This research showed differential gene expression and clinical indices depending on method of weight Classification [13].

The study aims to improve accuracy of Bioelectrical Impedance Analysis (BIA) prediction equations for estimating fat free mass (FFM) of the elderly by using nonlinear Back Propagation Artificial Neural Network (BPANN) model and to compare the predictive accuracy with the linear regression model by using energy dual X-ray absorptiometry (DXA) as reference method. When compared the performance of developed prediction equations for estimating reference $\mathrm{FFM}_{\mathrm{DXA}}$, the linear model has lower $r^{2}$ with a larger SD in predictive results than that of BP-ANN model, which indicated ANN model is more suitable for estimating FFM [14].

Although international interest in classifying subject health status according to adiposity is increasing, no accepted published ranges of percentage body fat currently exist. Empirically identified limits, population percentiles, and scores have all been suggested as means of setting percentage body fat guidelines, although each has major limitations. A convenient sample of 1626 adults with BMIs $\leq 35$ was evaluated. Independent percentage body fat predictor variables in multiple regression models included 1/BMI, sex, age, and ethnic group ( $R$ values from 0.74 to 0.92 and SEEs from 2.8 to $5.4 \%$ fat). The prediction formulas were then used to prepare provisional healthy percentage body fat ranges based on published BMI limits for underweight $(<18.5)$, overweight $(\geq 25)$, and obesity $(\geq 30)$. This proposed approach and initial findings provide the groundwork and stimulus for establishing international healthy body fat ranges [15].

Several studies have raised the suspicion that the body mass index (BMI) cut-off for overweight as defined by the WHO may not adequately reflect the actual overweight status. The present study looked at the relationship between BMI and body fat per cent (BF \%) / health risks (hypertension and type 2 diabetes) in male residents of Lucknow city, north India to evaluate the validity of BMI cut-off points for overweight. The study subjects showed higher body fat percentage and risk factors like hypertension and type 2 diabetes at normal BMI range proposed by the WHO. The cut-off for BMI was proposed to be 24.5 $\mathrm{kg} / \mathrm{m}^{2}$ for our study population. If overweight is regarded as an excess of body fat and not as an excess of weight (increased BMI), the cut-off points for overweight based on BMI would need to be lowered. However, the confidence of estimate of the BMI cut-off in the present study may be considered with the limitations of BI analysis studies [16].

Body composition assessment in patients with chronic renal failure is of paramount importance since studies have demonstrated the association of protein-energy malnutrition with an increased morbidity and mortality in this population. However, practical and sensible indicators of body compartments are still needed for clinical purposes. Thus, we aimed to evaluate the simple methods of skinfold thicknesses (SKF) and bioelectrical impedance analysis (BIA), using dual-energy X-ray absorptiometry (DEXA) as a reference method, for the assessment of body fat in patients on long-term haemodialysis therapy [17].

In this analysis of body composition such as Body Weight, BMI, Body Fat Percentage, Segmental Subcutaneous Fat \& Skeletal Muscle Percentage (Whole Body, Trunk, Legs and Arms), Resting Metabolism, Visceral Fat Level and Body Age is done by Bioelectrical impedance technique and results are interpreted and corresponding instructions for better health improvement is given.

\section{MATERIALS AND MethodS}

Body Composition Analysis of Teaching and Non Teaching Staff members of Brijlal Biyani Science College Amravati Maharashtra India Using Bioelectrical Impedance Analysis method is done. For ideal weight management and 
for a more accurate and precise body composition analysis full Body Sensing Technology Karada Scan Body Composition Monitor - HBF-375 as shown in figure 1 is used which measures body composition- weight, body fat percentage, visceral fat level, subcutaneous fat and skeletal muscle percentage, RM, BMI and Body age. The general principle behind bioelectrical impedance analysis is that two or more conductors are attached to a person's body and a small electric current is sent through the body. The resistance between the conductors will provide a measure of body fat between a pair of electrodes, since the resistance to electricity varies between adipose, muscular and skeletal tissue. Fat-free mass (muscle) is a good conductor as it contains a large amount of water (approximately 73\%) and electrolytes, while fat is anhydrous and a poor conductor of electric current. Each (bare) foot may be placed on an electrode, with the current sent up one leg, across the abdomen and down the other leg. There is little scope for technician error as such, but factors such as eating, drinking and exercising must be controlled since hydration level is an important source of error in determining the flow of the electric current to estimate body fat. The instructions for use of instruments typically recommended not making measurements soon after drinking or eating or exercising, or when dehydrated. Instruments require details such as sex and age to be entered, and use formulae taking these into account; for example, men and women store fat differently around the abdomen and thigh region.

BMI: Body mass index is defined as the individual's body mass divided by the square of his or her height. The formulae universally used in medicine produce a unit of measure of $\mathrm{kg} / \mathrm{m}^{2}$.

$$
\mathrm{BMI}=\text { Weight }(\mathrm{Kg}) /[\text { height }(\mathrm{m})]^{2}
$$

\section{Body fat percentage:}

Body fat percentage $=[$ Body fat Mass $(\mathrm{Kg}) /$ Body weight $(\mathrm{Kg})] \times 100$

Visceral Fat: Adipose tissue is one of the main types of connective tissue. In biology, adipose tissue or body fat or fat depot or just fat is loose connective tissue composed of adipocytes.

BMR (RMR): Basal metabolic rate (BMR), and the closely related resting metabolic rate (RMR), is the amount of energy expended daily by humans and other animals at rest. Rest is defined as existing in a neutrally temperate environment while in the postabsorptive state.

Skeletal muscle: Skeletal muscle is a form of striated muscle tissue existing throughout the human body, and which is under control of the somatic nervous system; that is to say, it is voluntarily controlled. It is one of three major muscle types, the others being cardiac and smooth muscle. As their name suggests, most skeletal muscles are attached to bones by bundles of collagen fibers known as tendons.

Biological (Real) Age: Biological age is how time and lifestyle have affected organs and cells compared to other people of chronological age. Factors of biological aging include changes in the physical structure of the body as well as changes in the performance of motor skills and sensory awareness. Chronological age is current age in years, calculated from birth date. Interpretation of Bioelectrical Impedance Analysis report is shown in Table I.

Body composition analysis of Teaching Staff members (Senior College \& Junior College) \& Non Teaching Staff members (Laboratory, Office \& Library) is shown in Table II

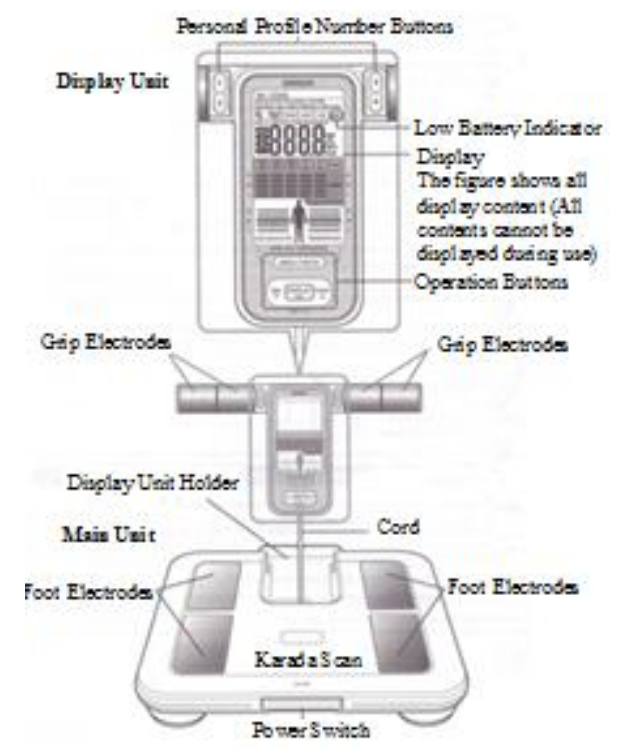

Fig 1. Omron Body Composition Scale Karada Scan HBF-375.

TABLE I: INTERPRETATION OF BIOELECTRICAL IMPEDANCE ANALYSIS

\begin{tabular}{|c|c|c|c|c|c|c|}
\hline \multicolumn{7}{|c|}{ Interpretation of Body Composition Report } \\
\hline \multirow{3}{*}{$\begin{array}{c}\text { Body } \\
\text { Fat \% }\end{array}$} & \multicolumn{2}{|c|}{ Gender } & Low & Normal & High & Very High \\
\hline & \multicolumn{2}{|c|}{ Female } & Up to 19.9 & $20-29.9$ & $30-34.9$ & $35 \&$ more \\
\hline & \multicolumn{2}{|c|}{ Male } & Up to 9.9 & $10-19.9$ & $20-24.9$ & $25 \&$ more \\
\hline & \multirow{2}{*}{\multicolumn{3}{|c|}{$\begin{array}{c}\text { Trunk Fat } \\
\%\end{array}$}} & Normal & High & Very high \\
\hline & & & & $<15$ & $16-18$ & $18+$ \\
\hline & \multirow{2}{*}{\multicolumn{3}{|c|}{ Visceral Fat \% }} & 0 (Normal) & $+($ High $)$ & $++($ Very High $)$ \\
\hline & & & & $0.5-9.5$ & $10.0-14.5$ & $15.0-30.0$ \\
\hline \multirow{7}{*}{$\begin{array}{c}\text { Skeletal } \\
\text { Muscle } \\
\%\end{array}$} & Gender & Age & $-($ Low $)$ & 0 (Normal) & $+($ High $)$ & $++($ Very High $)$ \\
\hline & \multirow{3}{*}{ Female } & $18-39$ & $<24.3$ & $24.3-30.3$ & $30.4-35.3$ & $\geq 35.4$ \\
\hline & & $40-59$ & $<24.1$ & $24.1-30.1$ & $30.2-35.1$ & $\geq 35.2$ \\
\hline & & $60-80$ & $<23.9$ & 23.9-29.9 & $30.0-34.9$ & $\geq 35.0$ \\
\hline & \multirow{3}{*}{ Male } & $18-39$ & $<33.3$ & $33.3-39.3$ & $39.4-44.0$ & $\geq 44.1$ \\
\hline & & $40-59$ & $<33.1$ & $33.1-39.1$ & $39.2-43.8$ & $\geq 43.9$ \\
\hline & & $60-80$ & $<32.9$ & $32.9-38.9$ & $39.0-43.6$ & $\geq 43.7$ \\
\hline & \multirow{2}{*}{\multicolumn{2}{|c|}{ BMI }} & Under wt & Normal & Over wt & Obese \\
\hline & & & Up to18.4 & $18.5-24.9$ & $25-29.9$ & $30 \&$ more \\
\hline
\end{tabular}


TABLE II: Body Composition ANALYSIS OF TEACHING StAFF \& NON-TEACHING STAFF MEMBERS

\begin{tabular}{|c|c|c|c|c|c|c|c|c|c|c|c|c|c|c|c|c|c|}
\hline \multicolumn{18}{|c|}{ Teaching Staff members (Senior College) } \\
\hline \multirow[b]{2}{*}{$\begin{array}{l}Z_{z} \\
0 \\
\tilde{z} \\
\tilde{z}\end{array}$} & \multirow[b]{2}{*}{ 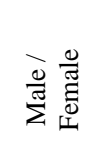 } & \multirow[b]{2}{*}{$\stackrel{8}{\&}$} & \multirow[b]{2}{*}{ 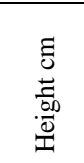 } & & & & subc & $\mathrm{Fi}$ & & & & reletal & Iuscle & & & & \\
\hline & & & & $\begin{array}{l}\frac{7}{000} \\
\frac{.00}{0} \\
3\end{array}$ & 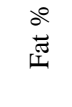 & $\begin{array}{l}\frac{0}{0} \overrightarrow{8} \\
\frac{0}{3}\end{array}$ & 兰 & 旁 & 范 & 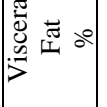 & $\begin{array}{l}\frac{0}{0} \\
\frac{2}{0} \\
\frac{1}{3}\end{array}$ & 兰 & 荎 & 峁 & 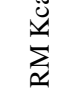 & $\sum_{\infty}$ & 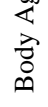 \\
\hline 1 & Male & 24 & 185 & 61 & 14.9 & 9.9 & 8.2 & 16.5 & 15.5 & 1.5 & 37.6 & 31.8 & 42.5 & 54.7 & 1506 & 17.8 & 18 \\
\hline 2 & Female & 25 & 152 & 44.2 & 25.4 & 20.9 & 17.0 & 37.0 & 32.2 & 2.0 & 26.7 & 22.4 & 32.0 & 37.6 & 1040 & 19.1 & 21 \\
\hline 3 & Female & 27 & 154.5 & 43.3 & 26.1 & 20.5 & 17.1 & 37.7 & 31.9 & 15 & 26.6 & 22.5 & 32.1 & 37.1 & 1024 & 18.1 & 20 \\
\hline 4 & Male & 28 & 168.6 & 79.6 & 30.1 & 21.0 & 19.2 & 30.5 & 30.7 & 12.5 & 29.5 & 21.3 & 35.9 & 46.9 & 1719 & 28.0 & 47 \\
\hline 5 & Female & 30 & 154 & 67.5 & 37.6 & 33.8 & 29.9 & 51.8 & 48.7 & 9.5 & 22.5 & 16.9 & 22.0 & 35.8 & 1349 & 28.5 & 49 \\
\hline 6 & Male & 33 & 173 & 54.3 & 23.3 & 15.2 & 12.9 & 22.5 & 22.3 & 2.5 & 33.5 & 26.3 & 40.2 & 50.7 & 1354 & 18.1 & 23 \\
\hline 7 & male & 36 & 163.5 & 74.7 & 37.8 & 33.5 & 29.7 & 51.6 & 47.8 & 9.0 & 23.0 & 16.9 & 21.0 & 36.1 & 1459 & 27.9 & 54 \\
\hline 8 & Male & 40 & 169 & 72.7 & 29.9 & 20.5 & 18.4 & 29.2 & 29.5 & 11.0 & 29.1 & 21.0 & 35.7 & 46.5 & 1608 & 25.5 & 49 \\
\hline 9 & ale & 43 & 173.5 & 89.2 & 31.9 & 22.4 & 20.6 & 30.7 & 31.3 & 16.5 & 28.3 & 19.9 & 34.5 & 45.7 & 1855 & 29.6 & 59 \\
\hline 10 & Male & 45 & 171 & 70.5 & 26.8 & 18.4 & 16.4 & 25.5 & 25.6 & 9.5 & 30.0 & 22.6 & 36.4 & 47.4 & 1588 & 24.1 & 48 \\
\hline 11 & Female & 48 & 148 & 38.7 & 31.6 & 22.9 & 20.6 & 42.2 & 32.4 & 2.0 & 22.8 & 18.5 & 28.7 & 32.4 & 931 & 17.7 & 36 \\
\hline 12 & ale & 48 & 173 & 74.8 & 31.2 & 21.2 & 19.1 & 29.9 & 30.4 & 11.5 & 28.5 & 20.2 & 35.2 & 45.9 & 1633 & 25.0 & 54 \\
\hline 13 & ale & 48 & 155 & 61.3 & 34.8 & 23.5 & 21.1 & 33.2 & 34.0 & 12.5 & 25.6 & 17.0 & 34.3 & 43.2 & 1398 & 25.5 & 53 \\
\hline 14 & ale & 49 & 176 & 87.1 & 36.9 & 33.1 & 29.2 & 48.7 & 43.9 & 10 & 24.5 & 16.8 & 20.2 & 38.0 & 1659 & 28.1 & 63 \\
\hline 15 & & 52 & 159 & 73.0 & 33.5 & 23.3 & 21.3 & 32.1 & 32.8 & 17.5 & 25.8 & 17.5 & 33.7 & 43.3 & 1582 & 28.9 & 62 \\
\hline 16 & ale & 59 & 172.5 & 77.4 & 29.1 & 20.1 & 18.1 & 26.3 & 26.7 & 13.5 & 28.5 & 20.8 & 34.7 & 45.9 & 1681 & 26.0 & 60 \\
\hline 17 & Male & 60 & 162.5 & 70.3 & 33.0 & 22.6 & 20.5 & 30.6 & 31.3 & 15.0 & 25.8 & 17.7 & 33.4 & 43.4 & 1542 & 26.6 & 63 \\
\hline & & & & & & Teac & $\operatorname{lng~St}$ & mem & $\mathrm{rs}(\mathrm{Ju}$ & or Colle & ege) & & & & & & \\
\hline 1 & Female & 25 & 151 & 35.2 & 19 & 15.0 & 10.9 & 29.8 & 25.3 & 0.5 & 28.4 & 24.8 & 36.0 & 38.2 & 913 & 15.4 & 18 \\
\hline 2 & Yale & 27 & 169 & 64.5 & 24.9 & 23.1 & 18.3 & 35.2 & 31.9 & 3.5 & 29.7 & 23.3 & 31.2 & 42.9 & 1377 & 22.6 & 31 \\
\hline 3 & male & 31 & 144.5 & 56.5 & 37.7 & 32.8 & 29.3 & 51.9 & 47.9 & 8.5 & 21.5 & 16.6 & 23.2 & 33.7 & 1180 & 27.1 & 45 \\
\hline 4 & Male & 36 & 165 & 66.2 & 32.0 & 28.0 & 24.1 & 43.9 & 39.2 & 6.0 & 25.5 & 19.5 & 25.9 & 38.0 & 1362 & 24.3 & 45 \\
\hline 5 & Female & 37 & 154 & 65.8 & 35.9 & 32.4 & 28.3 & 48.6 & 44.8 & 9.5 & 23.2 & 17.4 & 23.2 & 36.5 & 1332 & 27.7 & 52 \\
\hline 6 & Female & 39 & 159 & 72.6 & 41.3 & 35.8 & 32.5 & 56.8 & 53.0 & 10.5 & 21.1 & 15.4 & 19.3 & 33.8 & 1406 & 28.7 & 58 \\
\hline 7 & male & 39 & 56 & 53.2 & 33.3 & 26.9 & 23.8 & 5.7 & 39.1 & 4.0 & 23.8 & 18.8 & 26.7 & 36.2 & 1151 & 21.9 & 43 \\
\hline 8 & male & 39 & 52 & 54.5 & 34.7 & 28.8 & 25.6 & 47.4 & 41.3 & 5.5 & 23.0 & 18.0 & 25.4 & 35.5 & 1164 & 23.6 & 46 \\
\hline 9 & Female & 39 & 158 & 43.7 & 26.5 & 20.3 & 17.0 & 37.5 & 30.5 & 1.5 & 26.2 & 21.8 & 31.5 & 36.3 & 1028 & 17.5 & 28 \\
\hline 10 & & 40 & 2.5 & 82.8 & 33.3 & 1.1 & 5.6 & & 39.4 & 8.5 & & 18.9 & 23.9 & 40.9 & 1623 & 27.8 & 53 \\
\hline 11 & & 44 & 159 & 60.0 & 24.4 & 16.8 & 14.9 & 23.2 & 23.2 & 9.5 & 29.6 & 23.0 & 36.2 & 47.0 & 1424 & 23.7 & 44 \\
\hline 12 & Female & 44 & 145 & 48.6 & 37.1 & 29.6 & 27.0 & 49.7 & 42.2 & 6.0 & 21.2 & 16.5 & 24.6 & 33.0 & 1064 & 23.1 & 49 \\
\hline 13 & Female & 45 & 166.5 & 66.1 & 34.3 & 28.8 & 25.4 & 46.6 & 40.7 & 5.5 & 24.3 & 18.3 & 24.4 & 36.9 & 1347 & 23.8 & 52 \\
\hline 14 & & 51 & 50.5 & 73.6 & 31.5 & 21.9 & 20.1 & & 30.3 & 17.0 & & 18 & 33.8 & 44.3 & 1603 & 28.6 & 60 \\
\hline 15 & ale & 56 & 175 & 64.6 & 30.0 & 19.9 & 17.4 & 27.5 & 28.0 & 7.0 & 28.9 & 20.8 & 36.1 & 46.3 & 1479 & 21.1 & 48 \\
\hline & & & & & & Non 1 & - & 4 & , & abor & ory) & & & & & & \\
\hline 1 & & 25 & 168 & 62.4 & 23.9 & 16.2 & 14.2 & 24.4 & 24.1 & 6.0 & 32.6 & 25.4 & 39.0 & 49.9 & 1480 & 22.1 & 30 \\
\hline 2 & Male & 25 & & 55.1 & 29.2 & 19.5 & 17.2 & 29.0 & 29.2 & 6.0 & 30.5 & 22.3 & 38.0 & 47.8 & 1339 & 22.1 & 30 \\
\hline 3 & Male & 27 & 177 & 56.9 & 17.6 & 11.7 & 9.7 & 18.4 & 17.7 & 2.0 & 35.9 & 29.7 & 41.7 & 53.0 & 1421 & 18.2 & 18 \\
\hline 4 & Iale & 29 & 167 & 58.4 & 22.6 & 15.2 & 13.2 & 22.8 & 22.5 & 5.0 & 32.8 & 25.9 & 39.3 & 50.1 & 1419 & 20.9 & 28 \\
\hline 5 & & 29 & & 5.5 & 22.8 & 15.5 & & & 22.7 & 5.5 & & 26 & & 50 & 1536 & 21 & 32 \\
\hline 6 & & 30 & & 57.6 & 18.7 & 12.6 & 0.8 & & 18.6 & 3.5 & 34 & 28.3 & 40.5 & 51.7 & 1432 & 19.7 & 24 \\
\hline 7 & le & 33 & & 51.8 & & & & & & 4.0 & & 27.0 & 39.8 & 50.4 & 1318 & 19.5 & 25 \\
\hline 8 & & 33 & & & & & 18.9 & 29.9 & 30.2 & & & 20.5 & 35.3 & 45.8 & 1539 & 27.7 & 47 \\
\hline 9 & & 34 & 155.5 & 64.3 & 26.1 & 18.3 & 16.6 & 25.9 & 25.9 & 12.0 & 29.3 & 22.3 & 36.4 & 46.7 & 1487 & 26.6 & 44 \\
\hline 10 & & 35 & 169 & 91.7 & 30.6 & 22.0 & 20.5 & 29.2 & 29.6 & 18.5 & 29.0 & 20.8 & 34.4 & 46.4 & 1904 & 32.1 & 56 \\
\hline 11 & & 37 & & 8.7 & 27.8 & 19.1 & 17.1 & & 27.4 & 10.0 & & 22.1 & 36.6 & 47.1 & 1555 & 24.9 & 45 \\
\hline 12 & & 39 & & 5.9 & 0.1 & 3.0 & 0.8 & & 18.4 & 1.5 & & 1 & 4 & 49.4 & 1167 & 17.1 & 19 \\
\hline 13 & & 41 & 715 & 120 & 6.0 & 7.9 & 5.0 & 4.9 & 25.0 & 10.0 & 30.6 & 3 & 36.4 & 48.0 & 1618 & 24.5 & 46 \\
\hline 14 & & 43 & & & & & & & & 7.5 & & 3.9 & .0 & 48.2 & 1478 & 22.3 & 41 \\
\hline 15 & & 43 & & 78.7 & & & & & 22.9 & 16.5 & & 23.4 & 35.7 & 47.8 & 1726 & 29.8 & 54 \\
\hline 16 & & 44 & 162 & 49.7 & 26.7 & 17.4 & 15.0 & 24.2 & 24.5 & 40 & 29.9 & 22.6 & 37.8 & 47.3 & 1256 & 18.9 & 32 \\
\hline 17 & & 48 & 165.8 & 65.8 & 24.6 & 17.0 & 15.1 & 23.0 & 23.0 & 10.0 & 30.1 & 23.3 & 36.0 & 47.5 & 1519 & 23.9 & 47 \\
\hline 18 & & 51 & & 103 & & & & & & & & & & & & .0 & 73 \\
\hline 19 & & 53 & & 71.4 & 7.7 & & 6.9 & & 25.9 & & & 21.8 & & 46 & 1595 & 24 & 53 \\
\hline 20 & & 56 & 75 & & & 0.6 & & & 28.3 & 13.5 & & 0 & 4 & 45 & 1591 & 25.7 & 58 \\
\hline 21 & & 57 & & 66.3 & 26.8 & 18.1 & 16.0 & 24.4 & 24.7 & 9.0 & 29.2 & 22.0 & 35.9 & 46.6 & 1516 & 22.7 & 51 \\
\hline & & & & & & & & & & (OOII & & & & & & & \\
\hline 1 & male & 32 & 525 & 62.9 & 40.4 & 33.9 & 30.9 & 55.9 & 51.3 & 8.0 & 21.3 & 16.3 & 21.6 & 33.8 & 1267 & 26.7 & 49 \\
\hline 2 & & 32 & 2.5 & 62.0 & 23.2 & & & & & 5.0 & 32 & 25.7 & 39.0 & 1 & 1476 & 20.8 & 31 \\
\hline 3 & & 32 & & 64.2 & & 16.2 & & & 23.5 & 6.5 & 32.2 & 25.1 & 38.3 & 49.5 & 1507 & 22.2 & 35 \\
\hline 4 & & 35 & 162.5 & 9.3 & & 20.3 & 17.9 & 29.4 & 29.8 & 7.5 & 29.4 & 21.2 & 36.7 & 46.8 & 1398 & 22.5 & 39 \\
\hline 5 & & 38 & & & & & & & 28.2 & & & & 5.5 & 6.5 & 703 & 28.5 & 52 \\
\hline 6 & & 39 & & & & & & & & 6.0 & & 20.5 & 37.0 & 46.4 & 1326 & 21.0 & 37 \\
\hline 7 & & 39 & 157.5 & 65.8 & 28.0 & 19.4 & 17.6 & 27.3 & 27.5 & 12.5 & 28.5 & 21.2 & 35.4 & 46.0 & 1501 & 26.5 & 47 \\
\hline 8 & & 40 & 164 & 66.8 & 29.7 & 20.3 & 18.2 & 28.9 & 29.2 & 10.5 & 28.7 & 20.8 & 35.9 & 46.2 & 1514 & 24.8 & 47 \\
\hline 9 & & 42 & 170 & 68.8 & 26.7 & 18.2 & 16.2 & 25.6 & 25.7 & 9.0 & 30.2 & 22.8 & 36.6 & 47.6 & 1562 & 23.8 & 45 \\
\hline 10 & & 43 & 159 & 62.8 & 29.8 & 20.3 & 18.2 & 28.7 & 29.1 & 11.0 & 27.9 & 20.2 & 35.4 & 45.4 & 1446 & 24.8 & 48 \\
\hline 11 & & 43 & 169.2 & 82.9 & 30.8 & 21.6 & 19.8 & 29.5 & 30.0 & 16.0 & 28.4 & 20.3 & 34.7 & 45.9 & 1761 & 29.0 & 57 \\
\hline 12 & Male & 54 & 169.5 & 75.7 & 31.7 & 21.7 & 19.7 & 29.8 & 30.4 & 13.5 & 27.5 & 19.3 & 34.7 & 45.0 & 1640 & 26.3 & 59 \\
\hline
\end{tabular}




\begin{tabular}{|c|c|c|c|c|c|c|c|c|c|c|c|c|c|c|c|c|c|}
\hline \multicolumn{18}{|c|}{ Non Teaching Staff members (Library) } \\
\hline 1 & Male & 36 & 168.5 & 70.3 & 26.9 & 18.5 & 16.6 & 26.4 & 26.5 & 9.5 & 30.4 & 22.9 & 37.3 & 47.7 & 1587 & 24.8 & 44 \\
\hline 2 & Male & 40 & 161 & 63.3 & 25.8 & 17.8 & 15.9 & 24.9 & 25.0 & 10.0 & 29.7 & 22.7 & 36.6 & 47.1 & 1474 & 24.4 & 44 \\
\hline 3 & Male & 44 & 163.5 & 68.6 & 30.3 & 28.1 & 23.5 & 40.1 & 35.6 & 7.0 & 27.3 & 20.3 & 27.5 & 41.1 & 1414 & 25.7 & 50 \\
\hline 4 & Male & 50 & 167 & 74.4 & 27.8 & 19.4 & 17.6 & 25.7 & 26.0 & 13.5 & 29.0 & 21.5 & 35.4 & 46.4 & 1640 & 26.7 & 55 \\
\hline
\end{tabular}

\section{RESULT AND DISCUSSION}

The ideal BMI is 22. Maintaining an ideal weight can help prevent obesity or weight loss and other diseases, and lead a longer life. The ideal weight for BMI of 22 is calculated as follows.

$$
\text { Ideal Weight }(\mathrm{Kg})=22 \times[\text { height }(\mathrm{m})]^{2}
$$

However this method of ideal weight calculation may not be applicable for professional athletes and body builders, who have higher muscles ratio in their bodies.

Correct Weight Reduction: If we lose weight by going on a diet instead of doing exercise and neglecting nutrition balance, even if our weight is reduced, resting metabolism will decrease as muscle (Skeletal muscle) decreases and we are more likely to become fatter. We should build up non-fat physique by increasing skeletal muscle and improving resting metabolism.

Interpretation of body composition analysis report of teaching Staff members (Senior College \& Junior College) $\&$ Non Teaching Staff members (Laboratory, Office \& Library) is shown in Table III.

TABLE III: INTERPRETATION OF BODY COMPOSITION ANALYSIS REPORT OF TEACHING \& NON-TEACHING STAFF MEMBERS

\begin{tabular}{|c|c|c|c|c|c|c|c|c|c|c|c|}
\hline \multicolumn{12}{|c|}{ Teaching Staff members (Senior College) } \\
\hline 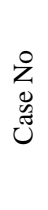 & 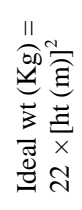 & 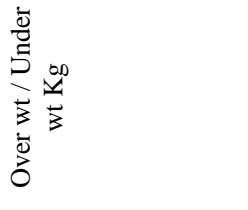 & 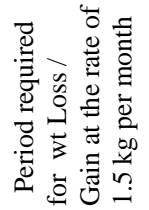 & 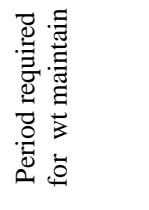 & $\begin{array}{l}\text { be } \\
\text { त्ञ }\end{array}$ & 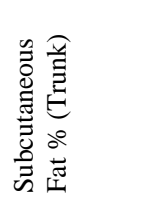 & 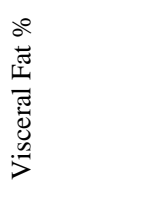 & 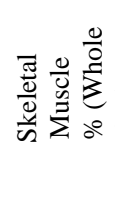 & 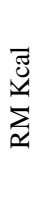 & $\sum_{\infty}$ & 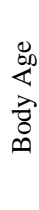 \\
\hline 1 & 75.3 & $\uparrow 14.3 \mathrm{Kg}$ Under wt & 9.5 Month & 9.5 Month & Normal & Normal & Normal & Normal & $\uparrow$ & $\uparrow$ Under wt & $\uparrow$ \\
\hline 2 & 50.8 & $\uparrow 6.6 \mathrm{Kg}$ Under wt & 4 Month & 4 Month & Normal & $\downarrow$ High & Normal & Normal & $\uparrow$ & Normal & $\uparrow$ \\
\hline 3 & 52.5 & $\uparrow 9.2 \mathrm{Kg}$ Under wt & 6 Month & 6 Month & Normal & $\downarrow$ High & $\downarrow$ Very High & Normal & $\uparrow$ & $\uparrow$ Under wt & $\uparrow$ \\
\hline 4 & 62.5 & $\downarrow 17.1 \mathrm{Kg}$ Over wt & 11.5 Month & 11.5 Month & $\downarrow$ Very High & $\downarrow$ Very high & $\downarrow$ High & $\uparrow$ Low & $\uparrow$ & $\downarrow$ Over wt & $\downarrow$ \\
\hline 5 & 52.2 & $\downarrow 15.3 \mathrm{Kg}$ Over wt & 10 Month & 10 Month & $\downarrow$ Very High & $\downarrow$ Very High & Normal & $\uparrow$ Low & $\uparrow$ & $\downarrow$ Over wt & $\downarrow$ \\
\hline 6 & 65.8 & $\uparrow 11.5 \mathrm{Kg}$ Under wt & 8 Month & 8 Month & $\downarrow$ High & Normal & Normal & Normal & $\uparrow$ & $\uparrow$ Under wt & $\uparrow$ \\
\hline 7 & 58.8 & $\downarrow 15.9 \mathrm{Kg}$ Over wt & 11 Month & 11 Month & $\downarrow$ Very High & $\downarrow$ Very High & Normal & $\uparrow$ Low & $\uparrow$ & $\downarrow$ Over wt & $\downarrow$ \\
\hline 8 & 62.8 & $\downarrow 9.9 \mathrm{Kg}$ Over wt & 6.5 Month & 6.5 Month & $\downarrow$ Very High & $\downarrow$ Very High & $\downarrow$ High & $\uparrow$ Low & $\uparrow$ & Over wt & $\downarrow$ \\
\hline 9 & 66.2 & $\downarrow 23 \mathrm{Kg}$ Over wt & 15 Month & 15 Month & $\downarrow$ Very High & $\downarrow$ Very High & $\downarrow$ Very High & $\uparrow$ Low & $\uparrow$ & $\downarrow$ Over wt & $\downarrow$ \\
\hline 12 & 65.8 & $\downarrow 9 \mathrm{Kg}$ Over wt & 6 Month & 6 Month & $\downarrow$ Very High & $\downarrow$ Very High & $\downarrow$ High & $\uparrow$ Low & $\uparrow$ & $\downarrow$ Over wt & $\downarrow$ \\
\hline 13 & 52.9 & $\downarrow 8.4 \mathrm{Kg}$ Over wt & 6 Month & 6 Month & $\downarrow$ Very High & $\downarrow$ Very High & $\downarrow$ High & $\uparrow$ Low & $\uparrow$ & $\downarrow$ Over wt & $\downarrow$ \\
\hline 14 & 68.1 & $\downarrow 19 \mathrm{Kg}$ Over wt & 13 Month & 13 Month & $\downarrow$ Very High & $\downarrow$ Very High & $\downarrow$ High & $\uparrow$ Low & $\uparrow$ & $\downarrow$ Over wt & $\downarrow$ \\
\hline 15 & 55.6 & $\downarrow 17.4 \mathrm{Kg}$ Over wt & 12 Month & 12 Month & $\downarrow$ Very High & $\downarrow$ Very High & $\downarrow$ Very High & $\uparrow$ Low & $\uparrow$ & $\downarrow$ Over wt & $\downarrow$ \\
\hline 16 & 65.5 & $\downarrow 11.9 \mathrm{Kg}$ Over wt & 8 Month & 8 Month & Normal & $\downarrow$ Very High & $\downarrow$ High & $\uparrow$ Low & $\uparrow$ & $\downarrow$ Over wt & $\downarrow$ \\
\hline 17 & 58.1 & $\downarrow 12.2 \mathrm{Kg}$ Over wt & 8 Month & 8 Month & $\downarrow$ Very High & $\downarrow$ Very High & Very High & $\uparrow$ Low & $\uparrow$ & $\downarrow$ Over wt & $\downarrow$ \\
\hline \multicolumn{12}{|c|}{ Teaching Staff members (Junior College) } \\
\hline 1 & 50.2 & $\uparrow 15 \mathrm{Kg}$ Under wt & 10 Month & 10 Month & $\uparrow$ Low & Normal & Normal & Normal & $\uparrow$ & $\uparrow$ Under wt & $\uparrow$ \\
\hline 2 & 66.8 & $\downarrow 1.7 \mathrm{Kg}$ Over wt & 1 Month & 1 Month & $\downarrow$ High & $\downarrow$ Very High & Normal & $\uparrow$ Low & $\uparrow$ & Normal & $\downarrow$ \\
\hline 3 & 45.9 & $\downarrow 10.6 \mathrm{Kg}$ Over wt & 7 Month & 7 Month & $\downarrow$ Very High & $\downarrow$ Very high & Normal & $\uparrow$ Low & $\uparrow$ & $\downarrow$ Over wt & $\downarrow$ \\
\hline 4 & 59.9 & $\downarrow 6.3 \mathrm{Kg}$ Over wt & 4 Month & 4 Month & $\downarrow$ Very High & $\downarrow$ Very high & Normal & $\uparrow$ Low & $\uparrow$ & Normal & $\downarrow$ \\
\hline 5 & 52.2 & $\downarrow 13.6 \mathrm{Kg}$ Over wt & 9 Month & 9 Month & $\downarrow$ Very High & $\downarrow$ Very High & Normal & $\uparrow$ Low & $\uparrow$ & $\downarrow$ Over wt & $\downarrow$ \\
\hline 6 & 55.6 & $\downarrow 17 \mathrm{Kg}$ Over wt & 11 Month & 11 Month & $\downarrow$ Very High & $\downarrow$ Very High & $\downarrow$ High & $\uparrow$ Low & $\uparrow$ & $\downarrow$ Over wt & $\downarrow$ \\
\hline 7 & 53.5 & $\uparrow 0.3 \mathrm{Kg}$ Under wt & 8 Days & 8 Days & $\downarrow$ High & $\downarrow$ Very high & Normal & $\uparrow$ Low & $\uparrow$ & Normal & $\downarrow$ \\
\hline 8 & 50.8 & $\downarrow 3.7 \mathrm{Kg}$ Over wt & 2.5 Month & 2.5 Month & $\downarrow$ High & $\downarrow$ Very High & Normal & $\uparrow$ Low & $\uparrow$ & Normal & $\downarrow$ \\
\hline 12 & 46.3 & $\downarrow 2.3 \mathrm{Kg}$ Over wt & 1.5 Month & 1.5 Month & $\downarrow$ Very High & $\downarrow$ Very High & Normal & $\uparrow$ Low & $\uparrow$ & Normal & $\downarrow$ \\
\hline 13 & 61.0 & $\downarrow 5.1 \mathrm{Kg}$ Over wt & 3.5 Month & 3.5 Month & $\downarrow$ High & $\downarrow$ Very High & Normal & Normal & $\uparrow$ & Normal & $\downarrow$ \\
\hline 14 & 56.7 & $\downarrow 16.9 \mathrm{Kg}$ Over wt & 11 Month & 11 Month & $\downarrow$ Very High & $\downarrow$ Very High & $\downarrow$ Very High & $\uparrow$ Low & $\uparrow$ & $\downarrow$ Over wt & $\downarrow$ \\
\hline 15 & 67.4 & $\uparrow 2.8 \mathrm{Kg}$ Under wt & 2 Month & 2 Month & $\downarrow$ Very High & $\downarrow$ High & Normal & $\uparrow$ Low & $\uparrow$ & Normal & $\uparrow$ \\
\hline \multicolumn{12}{|c|}{ Non Teaching Staff members (Laboratory) } \\
\hline 1 & 62.1 & $\downarrow 0.3 \mathrm{Kg}$ Over wt & 7 days & 7 days & $\downarrow$ High & Normal & Normal & $\uparrow$ Low & $\uparrow$ & Normal & $\downarrow$ \\
\hline 2 & 54.9 & $\downarrow 0.2 \mathrm{Kg}$ Over wt & 7 days & 7 days & $\downarrow$ Very High & $\downarrow$ High & Normal & $\uparrow$ Low & $\uparrow$ & Normal & $\downarrow$ \\
\hline 3 & 68.9 & $\uparrow 12 \mathrm{Kg}$ Under wt & 8 Month & 8 Month & Normal & Normal & Normal & Normal & $\uparrow$ & $\uparrow$ Under wt & $\uparrow$ \\
\hline 4 & 61.4 & $\uparrow 3 \mathrm{Kg}$ Under wt & 2 Month & 2 Month & $\downarrow$ High & Normal & Normal & $\uparrow$ Low & $\uparrow$ & Normal & $\uparrow$ \\
\hline 5 & 66.6 & $\uparrow 1.1 \mathrm{Kg}$ Under wt & 1 Month & 1 Month & $\downarrow$ High & Normal & Normal & $\uparrow$ Low & $\uparrow$ & Normal & $\downarrow$ \\
\hline 6 & 64.3 & $\uparrow 6.7 \mathrm{Kg}$ Under wt & 4.5 Month & 4.5 Month & $\uparrow$ Low & Normal & Normal & Normal & $\uparrow$ & Normal & $\uparrow$ \\
\hline 7 & 58.5 & $\uparrow 6.7 \mathrm{Kg}$ Under wt & 4.5 Month & 4.5 Month & Normal & Normal & Normal & $\uparrow$ Low & $\uparrow$ & Normal & $\uparrow$ \\
\hline 8 & 54.6 & $\downarrow 14 \mathrm{Kg}$ Over wt & 9 Month & 9 Month & Normal & $\downarrow$ Very High & $\downarrow$ High & $\uparrow$ Low & $\uparrow$ & $\downarrow$ Over wt & $\downarrow$ \\
\hline 9 & 53.2 & $\downarrow 11.1 \mathrm{Kg}$ Over wt & 7.5 Month & 7.5 Month & Normal & $\downarrow$ Very High & $\downarrow$ High & $\uparrow$ Low & $\uparrow$ & $\downarrow$ Over wt & $\downarrow$ \\
\hline 10 & 62.8 & $\downarrow 28.8 \mathrm{Kg}$ Over wt & 19 Month & 19 Month & $\downarrow$ Very High & $\downarrow$ Very High & $\downarrow$ Very High & $\uparrow$ Low & $\uparrow$ & $\downarrow$ Obese & \\
\hline
\end{tabular}




\begin{tabular}{|c|c|c|c|c|c|c|c|c|c|c|c|}
\hline 11 & 60.6 & $\downarrow 8.1 \mathrm{Kg}$ Over wt & 5.5 Month & 5.5 Month & $\downarrow$ Very High & $\downarrow$ High & $\downarrow$ High & $\uparrow$ Low & $\uparrow$ & Normal & $\downarrow$ \\
\hline 12 & 55.3 & $\uparrow 12.4 \mathrm{Kg}$ Under wt & 8 Month & 8 Month & Normal & Normal & Normal & $\uparrow$ Low & $\uparrow$ & $\uparrow$ Under wt & $\uparrow$ \\
\hline 13 & 64.7 & $\downarrow 7.3 \mathrm{Kg}$ Over wt & 5 Month & 5 Month & Very High & $\downarrow$ High & $\downarrow$ High & $\uparrow$ Low & $\uparrow$ & Normal & \\
\hline 14 & 62.1 & $\downarrow 0.9$ Kg Over wt & 15 Days & 15 Days & Very High & Normal & Normal & $\uparrow$ Low & $\uparrow$ & Normal & $\uparrow$ \\
\hline 15 & 58.1 & $\downarrow 20.6 \mathrm{Kg}$ Over wt & 14 Month & 14 Month & Very High & $\downarrow$ High & Very High & $\uparrow$ Low & $\uparrow$ & $\downarrow$ Over wt & $\downarrow$ \\
\hline 16 & 57.7 & $\uparrow 8 \mathrm{Kg}$ Under wt & 5 Month & 5 Month & $\downarrow$ Very High & Normal & Very High & $\uparrow$ Low & $\uparrow$ & Normal & $\uparrow$ \\
\hline 17 & 60.5 & $\downarrow 5.3 \mathrm{Kg}$ Over wt & 3.5 Month & 3.5 Month & $\downarrow$ High & Normal & $\downarrow$ High & $\uparrow$ Low & $\uparrow$ & Normal & $\uparrow$ \\
\hline 18 & 62.8 & $\downarrow 40 \mathrm{Kg}$ Over wt & 27 Month & 27 Month & $\downarrow$ Very High & Very High & Very High & $\uparrow$ Low & $\uparrow$ & $\downarrow$ Obese & $\downarrow$ \\
\hline 19 & 65.1 & $\downarrow 6.3 \mathrm{Kg}$ Over wt & 4 Month & 4 Month & $\downarrow$ Very High & $\downarrow$ High & $\downarrow$ High & $\uparrow$ Low & $\uparrow$ & Normal & - \\
\hline 20 & 61.7 & $\downarrow 10.5 \mathrm{Kg}$ Over wt & 7 Month & 7 Month & Very High & Very High & High & $\uparrow$ Low & $\uparrow$ & Over wt & \\
\hline 21 & 64.3 & $\downarrow 2$ Kg Over wt & 1 Month & 1 Month & Very High & $\downarrow$ High & Normal & $\uparrow$ Low & $\uparrow$ & Normal & $\uparrow$ \\
\hline \multicolumn{12}{|c|}{ Non Teaching Staff members (Office) } \\
\hline 1 & 51.8 & $\downarrow 11.1 \mathrm{Kg}$ Over wt & 7.5 Month & 7.5 Month & $\downarrow$ Very High & $\downarrow$ Very High & Normal & $\uparrow$ Low & $\uparrow$ & Over wt & \\
\hline 2 & 65.5 & $\uparrow 3.5 \mathrm{Kg}$ Under wt & 2 Month & 2 Month & $\downarrow$ High & Normal & Normal & $\uparrow$ Low & $\uparrow$ & Normal & $\uparrow$ \\
\hline 3 & 63.6 & $\downarrow 0.6 \mathrm{Kg}$ Over wt & 15 Days & 15 Days & $\downarrow$ High & Normal & Normal & $\uparrow$ Low & $\uparrow$ & Normal & \\
\hline 4 & 58.1 & $\downarrow 1.2 \mathrm{Kg}$ Over wt & 1 Month & 1 Month & Very High & $\downarrow$ High & Normal & $\uparrow$ Low & $\uparrow$ & Normal & \\
\hline 5 & 60.6 & $\downarrow 17.9 \mathrm{Kg}$ Over wt & 12 Month & 12 Month & Very High & Very High & $\downarrow$ High & $\uparrow$ Low & $\uparrow$ & Over wt & \\
\hline 6 & 57.7 & $\uparrow 2.7 \mathrm{Kg}$ Under wt & 2 Month & 2 Month & Very High & Very High & Normal & $\uparrow$ Low & $\uparrow$ & Normal & $\uparrow$ \\
\hline 7 & 54.6 & $\downarrow 11.2 \mathrm{Kg}$ Over wt & 7.5 Month & 7.5 Month & Very High & Very High & $\downarrow$ High & $\uparrow$ Low & $\uparrow$ & $\downarrow$ Over wt & \\
\hline 8 & 59.2 & $\downarrow 7.6 \mathrm{Kg}$ Over wt & 5 Month & 5 Month & $\downarrow$ Very High & Very High & High & $\uparrow$ Low & $\uparrow$ & Normal & \\
\hline 9 & 63.6 & $\downarrow 5.2 \mathrm{Kg}$ Over wt & 3.5 Month & 3.5 Month & $\downarrow$ Very High & $\downarrow$ High & Normal & $\uparrow$ Low & $\uparrow$ & Normal & \\
\hline 10 & 55.6 & $\downarrow 7.2 \mathrm{Kg}$ Over wt & 5 Month & 5 Month & $\downarrow$ Very High & Very High & $\downarrow$ High & $\uparrow$ Low & $\uparrow$ & Normal & \\
\hline 11 & 63.0 & $\downarrow 19.9 \mathrm{Kg}$ Over wt & 13 Month & $13 \mathrm{Mo}$ & $\downarrow$ High & Very High & Very High & $\uparrow$ Low & $\uparrow$ & $\downarrow$ Over wt & \\
\hline 12 & 63.2 & $\downarrow 12.5 \mathrm{Kg}$ Over wt & 8 Month & 8 Month & $\downarrow$ Very High & $\downarrow$ Very High & $\downarrow$ High & $\uparrow$ Low & $\uparrow$ & $\downarrow$ Over wt & \\
\hline \multicolumn{12}{|c|}{ Non Teaching Staff members (Library) } \\
\hline 1 & 62.5 & $\downarrow 7.8 \mathrm{Kg}$ Over wt & 5 Month & 5 Month & $\downarrow$ Very High & $\downarrow$ High & Normal & Normal & $\uparrow$ & Normal & \\
\hline 2 & 57.0 & $\downarrow 6.3 \mathrm{Kg}$ Over wt & 4 Month & 4 Month & $\downarrow$ Very High & Normal & $\downarrow$ High & $\uparrow$ Low & $\uparrow$ & Normal & \\
\hline 3 & 58.8 & $\downarrow 9.8 \mathrm{Kg}$ Over wt & 6.5 Month & 6.5 Month & $\downarrow$ Very High & $\downarrow$ Very High & Normal & $\uparrow$ Low & $\uparrow$ & $\downarrow$ Over wt & \\
\hline 4 & 61.4 & $\downarrow 13 \mathrm{Kg}$ Over wt & 9 Month & 9 Month & $\downarrow$ Very High & $\downarrow$ High & $\downarrow$ High & $\uparrow$ Low & $\uparrow$ & Over wt & \\
\hline
\end{tabular}

In Table III sign $\uparrow$ indicates corresponding value should increase and sign $\downarrow$ indicates corresponding value should decrease during weight loss or weight gain programme.

Interpretation of body composition analysis report of Teaching Staff members (Senior College) shows that overweight person is $70.6 \%$ while that of underweight person is $29.4 \%$ and normal person is $0 \%$.

Interpretation of body composition analysis report of Teaching Staff members (Junior College) shows that overweight person is $73.3 \%$ while that of underweight person is $20.0 \%$ and normal person is $6.7 \%$.

Interpretation of body composition analysis report of Non Teaching Staff members (Laboratory) shows that overweight person is $52.4 \%$ while that of underweight person is $42.9 \%$ and normal person is $4.8 \%$.

Interpretation of body composition analysis report of Non Teaching Staff members (Office) shows that overweight person is $76.9 \%$ while that of underweight person is $16.7 \%$ and normal person is $0 \%$.

Interpretation of body composition analysis report of Non Teaching Staff members (Library) shows that overweight person is $100 \%$ while that of underweight person is $0 \%$ and normal person is $0 \%$.

\section{CONCLUSION}

In Teaching Staff members (Senior College) case number 1 - RM has to be increased so that BMI and body age can be increased and has to undergo 9.5 month weight gain (at the rate of $1.5 \mathrm{~kg}$ per month) programme to increase 14.3 $\mathrm{kg}$ weight. And has to undergo 9.5 month weight maintain programme.

In case number 2 - Subcutaneous trunk Fat $\%$ has to be decreased and RM has to be increased so that body age can be increased. And has to undergo 4 month weight gain programme to increase $6.6 \mathrm{Kg}$ weight. And has to undergo 4 month weight maintain programme.

In case number 3 - Subcutaneous trunk Fat \% and visceral fat $\%$ has to be decreased and RM has to be increased so that BMI and body age can be increased. And has to undergo 6 month weight gain programme to increase $9.2 \mathrm{Kg}$ weight. And has to undergo 6 month weight maintain programme.

In case number 4 - Fat $\%$, subcutaneous trunk Fat $\%$ and visceral fat $\%$ has to be decreased and whole body skeletal muscle \% and RM has to be increased so that BMI and body age can be decreased. And has to undergo 11.5 month weight loss programme to decrease $17.1 \mathrm{Kg}$ weight. And has to undergo 11.5 month weight maintain programme.

In case number 5 - Fat $\%$ and Subcutaneous trunk Fat $\%$ has to be decreased and whole body skeletal muscle $\%$ and $\mathrm{RM}$ has to be increased so that BMI and body age can be decreased. And has to undergo 10 month weight loss programme to decrease $15.3 \mathrm{Kg}$ weight. And has to undergo 10 month weight maintain programme.

Likewise conclusion from other cases can be drawn.

\section{REFERENCES}

[1] U. G. Kyle et al., "Bioelectrical impedance analysis-part I: review of principles and methods," Clinical Nutrition, vol. 23, no. 5, pp. 1226 43, June 2004

[2] Body fat scales review and compare, January 2010.

[3] H. C. Lukaski, W. W. Bolonchuk, C. B. Hall, and W. A. Siders, "Validation of tetrapolar bioelectrical impedance method to assess human body composition," J. Appl. Physiol., vol. 60, no. 4, pp. 1327-32, April 1986.

[4] F. Slinde and L. Rossander-Hulthén, "Bioelectrical impedance: Effect of 3 identical meals on diurnal impedance variation and calculation of body composition," Am. J. Clin. Nutr., vol. 74, no. 4, pp. 4748, October, 2001

[5] R. F. Kushner, R. Gudivaka, and D. A. Schoeller, "Clinical characteristics influencing bioelectrical impedance analysis measurements," Am. J. Clin. Nutr., vol. 64, 3 suppl, pp. 423S-427S, September 1996.

[6] M. Abu Khaled, M. J. McCutcheon, S. Reddy, P. L. Pearman, G. R. Hunter, and R. L. Weinsier, "Electrical impedance in assessing human body composition: the BIA method," Am. J. Clin. Nutr., vol. 47, no. 5, pp. 789-92, May 1988. 
[7] M. Dehghan and A. T. Merchant, "Is bioelectrical impedance accurate for use in large epidemiological studies?" Nutr J, vol. 7, pp. 26, 2008.

[8] A. C. Buchholz, C. Bartok, and D. A. Schoeller, "The validity of bioelectrical impedance models in clinical populations," Nutr Clin Pract, vol. 19, no. 5, pp. 433-46, October 2004.

[9] J. T. Peterson, W. E. S. Repovich, and C. R. Parascand, "Accuracy of consumer grade bioelectrical impedance analysis devices compared to air displacement plethysmography," Int J Exerc Sci, vol. 4, no. 3, pp. 176-184, 2011.

[10] G. Sun, C. R. French, G. R. Martin, B. Younghusband, R. C. Green, Y. G. Xie, M. Mathews, J. R. Barron, D. G. Fitzpatrick, W. Gulliver, and H. Zhang, "Comparison of multifrequency bioelectrical impedance analysis with dual-energy X-ray absorptiometry for assessment of percentage body fat in a large, healthy population," $\mathrm{Am}$ J Clin Nutr., vol. 81, no. 1, pp. 74-8, Jan. 2005.

[11] R. Roubenoff, "Applications of bioelectrical impedance analysis for body composition to epidemiologic studies," Am J Clin Nutr., vol. 64 , 3 supp, pp. 459S-462S, Sep. 1996.

[12] R. R. Biaggi, M. W. Vollman, M. A. Nies, C. E. Brener, P. J. Flakoll, D. K. Levenhagen, M. Sun, Z. Karabulut, and K. Y. Chen, "Comparison of air-displacement plethysmography with hydrostatic weighing and bioelectrical impedance analysis for the assessment of body composition in healthy adults," Am J Clin Nutr., vol. 69, no. 5, pp. 898-903, May 1999.

[13] V. L. Peterson1, A. C. Martino, A. Y. Del Valle-Pinero1, N. S. Patel, X. Zhao, and W. A. Henderson, "Bentham weight phenotype diagnostic test method: body mass index or body fat percent for gene expression," The Open Obesity Journal, 2012, vol. 4, 18-2218768237/12, 2012.

[14] K. C. Hsieh, Y. J. Chen, H. K. Lu, L. C. Lee, Y. C. Huang, and Y. Y. Chen, "The novel application of artificial neural network on bioelectrical impedance analysis to assess the body composition in elderly," Nutrition Journal, vol. 12, pp. 21, 2013.

[15] D. Gallagher, S. B. Heymsfield, M. Heo, S. A. Jebb, P. R. Murgatroyd, and Y. Sakamoto, "Healthy percentage body fat ranges: an approach for developing guidelines based on body mass index 1.2:3," The American Journal of Clinical Nutrition, Am J Clin Nutr, vol. 72, no. 3, pp. 694-70, September 2000.

[16] C. N. Kesavachandran, V. Bihari, and N. Mathur, "The normal range of body mass index with high body fat percentage among male residents of Lucknow city in north India," Indian J Med Res., vol. 135, no. 1, pp. 72-77, January 2012.

[17] M. A. Kamimura, C. M. Avesani, M. Cendoroglo, M. E. Canziani, S. A. Draibe, and L. Cuppari, "Comparison of skinfold thicknesses and bioelectrical impedance analysis with dual-energy X-ray absorptiometry for the assessment of body fat in patients on long-term haemodialysis therapy," Nephrol Dial Transplant, vol. 18, no. 1, pp. 101-5, Jan. 2003.

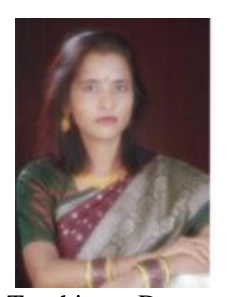

Swaroopa Rani N. Gupta was born on February 28, 1965 in Nagpur, Maharashtra, India. She got he S.S.C. in Somalwar High School, Khamla, Nagpur, Maharashtra, India. Maths, science, social science in 1981; H.S.S.C. in Hislop College, Nagpur, Maharashtra, India. Maths \& statistics, chemistry, biology in 1983; B.Sc. in Shivaji Science College, Nagpur, Maharashtra, India. Chemistry, zoology, microbiology in 1986; M.Sc. in Post Graduate

Teaching Department of Chemistry, Nagpur, Maharashtra, India. Chemistry Specialization: Analytical Chemistry in 1988; M.Phil. Post Graduate Teaching Department of Chemistry, Nagpur, Maharashtra, India. Coordination Chemistry, Polymer Chemistry in 1989; Ph.D. in Post Graduate Teaching Department of Chemistry, Nagpur, Maharashtra, IndiaChemistry in 1993.

She was now an associate professor in the Department of Chemistry, Brijlal Biyani Science College, Amravati, Maharashtra, India

Her research interests are in the field of chemistry especially analytical chemistry, environmental science and recent technologies connected to different field. Want to explore world through great research interest in all aspect of world problem. 